\title{
Control of Discrete 2-D Takagi-Sugeno Systems Via a Sum-Of-Squares Approach
}

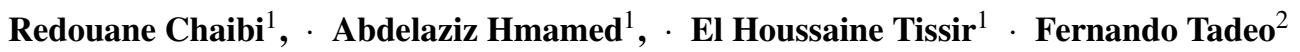

Received: date / Accepted: date

\begin{abstract}
The stabilization of Takagi-Sugeno (T-S) systems is solved here for the two-dimensional (2-D) polynomial discrete case, by using the sum of squares (SOS) approach. First, we provide a stabilization condition formulated in terms of Polynomial Multiple Lyapunov functions (PMLF). Then, a non-quadratic stabilization condition is developed by applying relaxed stabilization technique. Both conditions can be used for design, by solving them using numerical tools such as SOSTOOLS. A numerical example illustrates the effectiveness of the results.
\end{abstract}

Keywords discrete 2-D systems - Sum-of-Squares (SOS) · stabilization · Takagi-Sugeno systems.

\section{Introduction}

Two-dimensional (2-D) systems [1], [2], are drawning great attention in Systems Theory due to their extensive applications in practice, for instance, in multi-dimensional signal processing and transmission, and in thermal process. In fact, 2-D systems are very appropiate to model physical processes described by partial differential equations [3]. Moreover, 2-D techniques can also be applied as an analysis tool to solve complex control problems, for example, PI control of discrete linear repetitive processes [4], iterative learning

R. Chaibi, A. Hmamed, El H. Tissir

LESSI, Department of Physics Faculty of Sciences Dhar El Mehraz, B.P. 1796 Fes-Atlas Morocco

E-mail: c.redouane.chaibi@gmail.com,

elhoussaine.tissir@usmba.ac.ma,

hmamed_abdelaziz@yahoo.fr

F. Tadeo

Departamento de Ingenieria de Sistemas y Automatica and Instituto de Procesos Sostenibles, Universidad de Valladolid, 47005 Valladolid, Spain.

E-mail: small Fernando.Tadeo@uva.es control [5], and repetitive process control [2]. Several 2$\mathrm{D}$ models have been proposed, depending on the application [6]. The approach here is inspired by [7], where sufficient conditions for robust $H_{\infty}$ filtering were derived for 2-D discrete Roesser systems using homogenous polynomially parameter-dependent matrices of arbitrary degree, and in [8], that dealt with a parallel problem with time-varying delays.

These previous results focused on linear 2-D systems: we aim here to study the 2-D non-linear systems that can be represented using Takagi-Sugeno (T-S) models: these T$S$ models [9] are attracting a great deal of attention because they can effectively approximate a wide class of nonlinear systems, which can then be treated by adapting some linear systems techniques. For example, quadratic stabilization of T-S systems [10], [11] has been widely investigated based on a common quadratic Lyapunov function and the Parallel Distributed Compensation (PDC) [12], that unfortunately tends to give conservative conditions. Many other stability conditions for nonlinear systems have been investigated (see, for instance, [10], [13] and references therein). We emphasize here Multiple Lyapunov functions as they lead to good results in the sense that a common Lyapunov quadratic function may not exist but a multiple one exists (see [14], [15]), making possible to ensure stability for a wider class of nonlinear systems. Based on the T-S model, relaxed stabilization conditions are given in [16], by considering the information of some membership functions. Stability and stabilization conditions were derived for continuoustime T-S models in [17]. Various state-space models for 2D systems have been suggested in [18] to solve complex problems in different fields, for example, robust $H_{\infty}$ filtering of T-S systems, and stabilization of T-S systems with attenuation of stochastic perturbation. Relaxed stabilization conditions were developed in [19] by using non-quadratic stabilization conditions and a homogeneous polynomially 
parameter-dependent Lyapunov function. By using the basisdependent Lyapunov function approach and adding slack matrices, the $H_{\infty}$ filtering problem for 2-D T-S systems described by the Fornasini-Marchesini (FM) model was solved in [20]. Sufficient LMI conditions were also studied in [21] considering the effect of stochastic perturbations.

The techniques used here are instead based on a SumOf-Squares (SOS) approach [22], which makes possible to investigate the stability of polynomial systems for a range of problems that is wider than the more extensively used LMI approaches. SOS conditions can be numerically solved using off-the-shelf tools such as SOSTOOLS [22], [23]. More precisely, we use techniques based on those in [24], for polynomial T-S systems. SOS techniques have mainly been applied to 1-D continuous-time nonlinear systems: For example, the stability of polynomial systems with time-delay was presented in [25], using a novel polynomial Lyapunov-Krasovskii functional; Stability conditions for continuous-time polynomial systems were investigated in [26], by using polynomial Lyapunov functions; finally, we mention the effective technique for constructing Lyapunov functions for continuoustime multidimensional nonlinear systems in [27]. So far, few approaches have been proposed to deal with 2-D T-S system using SOS techniques: we can just cite [28], where a stabilization condition of the discrete 2-D T-S system based on polynomial Lyapunov functions has been provided. We must point out that the fact that in 2-D systems the information flows along two different directions makes controller synthesis for 2-D T-S systems more complex and challenging, especially when a polynomial model is considered, i.e., a T-S model whose consequent part is represented by a polynomial. Thus, this paper concentrates on designing the polynomial T-S control for nonlinear 2-D system that are characterized in the Roesser model; this is more challenging problem that parallel problems studied in the literature, such as those in [29].

Thus, this paper delivers an SOS-based methodology to solve the stabilization of Roesser-type polynomial discrete 2-D systems, whose consequent parts are depicted by polynomials. In this manner, the polynomial nonlinearities can be manipulated exactly and a large class of other nonlinearities can be treated by introducing auxiliary variables and constraints. We first derive a stabilization condition, based on polynomial multiple Lyapunov functions. Then, a nonquadratic stabilization condition is derived by using relaxation techniques. The proposed stabilization conditions are represented in terms of SOS and are numerically solved (partially symbolically) via SOSTOOLS [23]. We emphasize that the stabilization approach discussed in this paper is more general than existing LMI approaches to 2D T-S systems, thanks to the use of the Sum-Of-Squares approach.

The remainder of the paper is organized as follows: Section 2 provides the preliminaries and notation used through- out the paper; the main results are presented in Section 3; a numerical example is provided in Section 4 to demonstrate the effectiveness of the developed approach; finally, some conclusions are given in Section 5.

\section{Problem Formulation}

\section{Notation:}

- $\mathbb{R}$ : the set of real numbers;

- $\mathbb{Z}^{+}$: the set of nonnegative integers

- $\mathbb{C}^{1}$ : the set of complex numbers

- $I$ : identity matrix (of size specified by the context);

- $\lambda_{i}($.$) : i$ th eigenvalue;

- $*$ : represents a term that is induced by symmetry.

We consider here discrete nonlinear 2-D systems described as follows:

$x^{+}(k, l)=\mathscr{Z}(x(k, l))+\mathscr{S}(x(k, l)) u(k, l)$

$x^{h}(0, l)=f(l), \quad x^{v}(k, 0)=g(k)$

with

$x(k, l)=\left[\begin{array}{l}x^{h}(k, l) \\ x^{v}(k, l)\end{array}\right], x^{+}(k, l)=\left[\begin{array}{l}x^{h}(k+1, l) \\ x^{v}(k, l+1)\end{array}\right]$,

where $x^{h}(.) \in \mathbb{R}^{n_{1}}, x^{v}(.) \in \mathbb{R}^{n_{2}}$ are the horizontal and the vertical state, respectively; $u(.) \in \mathbb{R}^{m}$ is the control vector; $\mathscr{Z}($.$) and \mathscr{S}($.$) are nonlinear functions satisfying \mathscr{Z}, \mathscr{S}$ $\in \mathbb{C}^{1} ; k, l$ are two integers in $\mathbb{Z}^{+}$; finally, $f(l), g(k)$ are the boundary conditions along the horizontal and vertical directions, respectively.

The following equivalent discrete 2-D T-S system will be used, to represent the nonlinear Roesser system (1): Rule $i$ : IF $z_{1}(k, l)$ IS $M_{i 1}$ AND, $\ldots$, AND $z_{s}(k, l)$ IS $M_{i s}$ THEN

$$
\begin{gathered}
x^{+}(k, l)=A_{i}(x(k, l)) \hat{x}(x(k, l))+B_{i}(x(k, l)) u(k, l) \\
x^{h}(0, l)=f(l), \quad x^{v}(k, 0)=g(k)
\end{gathered}
$$

with

$A_{i}(x(k, l))=\left[\begin{array}{l}A_{11}^{i}(x(k, l)) A_{12}^{i}(x(k, l)) \\ A_{21}^{i}(x(k, l)) A_{22}^{i}(x(k, l))\end{array}\right]$,

$B_{i}(x(k, l))=\left[\begin{array}{l}B_{1}^{i}(x(k, l)) \\ B_{2}^{i}(x(k, l))\end{array}\right]$

where $i=1, \ldots, r, M_{i s}$ are the fuzzy sets, $z_{p}(k)$, for $p=$ $1, \ldots, s$, are the premise variables, $r$ is the number of IFTHEN rules, $\hat{x}(x(k, l))$ is a column vector whose entries are all monomials in $x(k, l)$ and $A_{i}(x(k, l)), B_{i}(x(k, l))$ are polynomial matrices in $x(k, l)$, with appropriate dimensions. 
These polynomial discrete 2-D T-S systems can also be expressed in a more compact form as follows

$$
\begin{aligned}
x^{+}(k, l) & =\sum_{i=1}^{r} h_{i}(z(k, l))\left\{A_{i}(x(k, l)) \hat{x}(x(k, l))\right. \\
& \left.+B_{i}(x(k, l)) u(k, l)\right\}
\end{aligned}
$$

where

$h_{i}(z(k, l))=\frac{w_{i}(z(k, l))}{\sum_{i=1}^{r} w_{i}(z(k, l))}, w_{i}(z(k, l))=\prod_{j=1}^{s} M_{i j}(z(k, l))$

where $M_{i j}(z(k, l))$ is the grade of membership of $z_{j}(k, l)$ in $M_{i j}$ and $w_{i}(z(k, l))$ represents the weight of the $i^{t h}$ rule. In this paper, we assume that $w_{i}(z(k, l)) \geq 0$, for $i=1,2, \ldots, r$, and $\sum_{i=1}^{r} w_{i}(z(k, l))>0$ for all $t$. Therefore, we get $h_{i}(z(k, l)) \geq$ 0 , for $i=1,2, \ldots, r$ and $\sum_{i=1}^{r} h_{i}(z(k, l))=1$ for all $t$.

Remark 1 The region of validity of this system is defined by $\mathscr{V}_{0}$ :

$\mathscr{V}_{0}=\left\{x(k) \in \mathbb{R}^{n} ;\left|L_{(N)} x(k)\right| \leq \eta_{(N)}\right\}$

where $\eta_{(N)}>0$ and $L_{(N)} \in \mathbb{R}^{1 \times n}$ for $N=1, \ldots \mathfrak{n}$ with $\mathfrak{n}$ representing the number of constraints that characterize the allowed region for the closed-loop system in the state space. For detailed discussions about the use of T-S models to represent exactly nonlinear systems inside a region $\mathscr{V}_{0}$ we can cite [35, 36]: although those results are for one dimensional systems, they can be directly extended to multidimensional systems, like the $2 D T-S$ in this paper.

Some stabilization conditions for the 2-D polynomial discrete systems in (4) will be later derived using the SOS approach, which is now introduced.

Lemma 1 [30] For two symmetric matrices $P>0$ and $Q>$ 0 , the inequality $A^{T} Q A-P<0$ holds, if there exist a matrix $G$ such that

$\left[\begin{array}{cc}P & * \\ G A & G+G^{T}-Q\end{array}\right]>0$

Definition 1 [22] A multivariate polynomial $f(x)$, for $x \in$ $\mathbb{R}^{N}$, is a SOS if there exist polynomials $f_{i}(x), i=1, \ldots, n$ such that

$f(x)=\sum_{i=1}^{n} f_{i}^{2}(x)$

This implies $f(x) \geq 0$ for any $x \in \mathbb{R}^{n}$.

Lemma 2 [31] Let $f(x)$ be a polynomial in $x \in \mathbb{R}^{n}$ of degree $2 d$. Let $Z(x)$ be a column vector whose entries are all monomial in $x$, with a degree no greater than $d$. Then, $f(x)$ is said to be SOS if and only if there exists a positive semi-definite matrix $Q$ such that

$f(x)=Z(x)^{T} Q Z(x)$

\section{Main Results}

To simplify the calculations, the following notations will be adopted:

$h_{i}=h_{i}(z(k, l)), \quad M_{z}(\tilde{x})=\sum_{i=1}^{r} h_{i} M_{i}(x(k, l))$,

$M_{z}^{-1}(\tilde{x})=\left(\sum_{i=1}^{r} h_{i} M_{i}(x(k, l))\right)^{-1}$

$K=\left\{k_{1}, k_{2}, \ldots, k_{n}\right\}$ denotes the set of row indices of $B_{i}(\tilde{x})$ whose corresponding row is equal to zeros; we then define $\tilde{x}=\left(x_{k_{1}}, x_{k_{2}}, \ldots, x_{k_{n}}\right)$. We will consider $T(x(k, l))$ that is a polynomial matrix defined by $\hat{x}(x(k, l))=T(x(k, l)) x(k, l)$.

3.1 Stabilization Conditions Via Polynomial Multiple Lyapunov function

In [33], a PDC control scheme and a polynomial Lyapunov function are proposed for 1-D to obtain less conservative stabilization conditions. In this paper we extend the PDC scheme used in the literature for 1-D T-S polynomial fuzzy systems to the 2-D systems studied in this paper. This 2-D controller can be expressed as follows

Rule $i$ : IF $z_{1}(k, l)$ IS $M_{i 1}$ AND, $\ldots$, AND $z_{s}(k, l)$ IS $M_{i s}$ THEN

$u(k, l)=F_{i}(\tilde{x}) \hat{x}(x(k, l))$

with $i=1, \ldots, r$, and $F_{i}(\tilde{x})$ polynomial matrices of appropriate dimensions to be determined. The overall 2-D controller can be represented by

$u(k, l)=\sum_{i=1}^{r} h_{i} F_{i}(\tilde{x}) \hat{x}(x(k, l))=F_{z}(\tilde{x}) \hat{x}(x(k, l))$

the closed-loop system is given by

$$
\begin{aligned}
x^{+}(k, l) & =\sum_{i=1}^{r} \sum_{j=1}^{r} h_{i} h_{j}\left\{A_{i}(\tilde{x})+B_{i}(\tilde{x}) F_{j}(\tilde{x})\right\} \hat{x}(x(k, l)) \\
& =\left(A_{z}(\tilde{x})+B_{z}(\tilde{x}) F_{z}(\tilde{x})\right) \hat{x}(x(k, l))
\end{aligned}
$$

Theorem 1 The polynomial T-S system (9) is asymptotically stable if there exists symmetric polynomial matrices $X_{1}^{j}(\tilde{x}) \in \mathbb{R}^{n_{1} \times n_{1}}, X_{2}^{j}(\tilde{x}) \in \mathbb{R}^{n_{2} \times n_{2}}$, and a polynomial matrices $K_{1}^{j}(\tilde{x}) \in \mathbb{R}^{m_{1} \times n_{1}}, K_{2}^{j}(\tilde{x}) \in \mathbb{R}^{m_{2} \times n_{2}}$, where $\varepsilon_{1}^{i}(\tilde{x})>0, \varepsilon_{2}^{i}(\tilde{x})>0$ for $(\tilde{x} \neq 0)$ and $\varepsilon_{1}^{i j}(\tilde{x}) \geq 0, \varepsilon_{2}^{i j}(\tilde{x}) \geq 0$ for all $\tilde{x}$ such that the following SOS holds:

$v_{1}^{T}\left(X_{1}^{i}(\tilde{x})-\varepsilon_{1}^{i}(\tilde{x}) I\right) v_{1} \quad$ is $\operatorname{SOS} i=1, \ldots, r$

$v_{1}^{T}\left(X_{2}^{i}(\tilde{x})-\varepsilon_{2}^{i}(\tilde{x}) I\right) v_{1} \quad$ is $\operatorname{SOS} i=1, \ldots, r$

$v_{2}^{T} \Omega_{i i}^{m n}(\tilde{x}) v_{2} \quad$ is $\operatorname{SOS} i, m, n=1, \ldots, r$

$v_{2}^{T}\left(\Omega_{i j}^{m n}(\tilde{x})+\Omega_{j i}^{m n}(\tilde{x})\right) v_{2}$ is $\operatorname{SOS}$ 
$i<j \quad i, j, m, n=1, \ldots, r$

with $v_{1}, v_{2}$ vectors of appropriate dimensions, that are independent of $x(k, l)$, and

$\Omega_{i j}^{m n}(\tilde{x})=\left[\begin{array}{cccc}\Omega_{i j 11}(\tilde{x}) & * & * & * \\ 0 & \Omega_{i j 22}(\tilde{x}) & * & * \\ \Omega_{i j 31}(\tilde{x}) & \Omega_{i j 32}(\tilde{x}) & \Omega_{i j 33}^{m}(\tilde{x}) & * \\ \Omega_{i j 41}(\tilde{x}) & \Omega_{i j 42}(\tilde{x}) & 0 & \Omega_{i j 44}^{n}(\tilde{x})\end{array}\right]$ $>0$

$\Omega_{i j 11}(\tilde{x})=X_{1}^{j}(\tilde{x})-\varepsilon_{1}^{i j}(\tilde{x}) I$

$\Omega_{i j 22}(\tilde{x})=X_{2}^{j}(\tilde{x})-\varepsilon_{2}^{i j}(\tilde{x}) I$

$\Omega_{i j 31}(\tilde{x})=T_{1}\left(\tilde{x}^{+}\right) A_{11}^{i}(\tilde{x}) X_{1}^{j}(\tilde{x})+T_{1}\left(\tilde{x}^{+}\right) B_{1}^{i}(\tilde{x}) K_{1}^{j}(\tilde{x})$

$\Omega_{i j 32}(\tilde{x})=T_{1}\left(\tilde{x}^{+}\right) A_{12}^{i}(\tilde{x}) X_{2}^{j}(\tilde{x})+T_{1}\left(\tilde{x}^{+}\right) B_{1}^{i}(\tilde{x}) K_{2}^{j}(\tilde{x})$

$\Omega_{i j 33}^{m}(\tilde{x})=X_{1}^{m}(x(k+1, l))-\varepsilon_{1}^{i j}(\tilde{x}) I$

$\Omega_{i j 41}(\tilde{x})=T_{2}\left(\tilde{x}^{+}\right) A_{21}^{i}(\tilde{x}) X_{1}^{j}(\tilde{x})+T_{2}\left(\tilde{x}^{+}\right) B_{2}^{i}(\tilde{x}) K_{1}^{j}(\tilde{x})$

$\Omega_{i j 42}(\tilde{x})=T_{2}\left(\tilde{x}^{+}\right) A_{22}^{i}(\tilde{x}) X_{2}^{j}(\tilde{x})+T_{2}\left(\tilde{x}^{+}\right) B_{2}^{i}(\tilde{x}) K_{2}^{j}(\tilde{x})$

$\Omega_{i j 44}^{n}(\tilde{x})=X_{2}^{n}(x(k, l+1))-\varepsilon_{2}^{i j}(\tilde{x}) I$

Proof Considering the following polynomial multiple Lyapunov function:

$V(x(k, l))=\hat{x}^{T}(x(k, l)) P_{z}(\tilde{x}) \hat{x}(x(k, l))$

where $P_{z}(\tilde{x})$ is a polynomial matrix in $x(k, l)$ such that

$P_{z}(\tilde{x})=\left[\begin{array}{cc}P_{1}^{z}(\tilde{x}) & 0 \\ 0 & P_{2}^{z}(\tilde{x})\end{array}\right]>0$

$\lambda_{\text {min }}\left(P_{z}(\tilde{x})\right)\|\hat{x}(x(k, l))\|_{2}^{2} \leq V(x(k, l)) \leq \lambda_{\max }\left(P_{z}(\tilde{x})\right)\|\hat{x}(x(k, l))\|_{2}^{2}$

where $\lambda_{\min }($.$) and \lambda_{\max }($.$) denote the minimum and max-$ imum eigenvalueS of a matrix, respectively.

Note that if $P_{z}(\tilde{x})$ is a constant matrix and $\hat{x}(x(k, l))=$ $x(k, l)$, then (15) reduces to the multiple Lyapunov function $x^{T}(k, l) P_{z} x(k, l)$ used in the literature: therefore $(15)$ is a more general representation, and will reduce conservatism in the developed conditions.

The variation of (15) is given by

$$
\begin{aligned}
\Delta V(x(k, l)) & =\hat{x}^{+T}(x(k, l)) P_{z}\left(\tilde{x}^{+}\right) \hat{x}^{+}(x(k, l)) \\
& -\hat{x}^{T}(x(k, l)) P_{z}(\tilde{x}) \hat{x}(x(k, l)) \\
& =\hat{x}^{T}(x(k, l))\left[\left(\tilde{A}_{z}(\tilde{x})+\tilde{B}_{z}(\tilde{x}) F_{z}(\tilde{x})\right)^{T} P_{z}\left(\tilde{x}^{+}\right)\right. \\
& \left.\times\left(\tilde{A}_{z}(\tilde{x})+\tilde{B}_{z}(\tilde{x}) F_{z}(\tilde{x})\right)-P_{z}(\tilde{x})\right] \hat{x}(x(k, l)) \\
& =-\hat{x}^{T}(x(k, l)) Q_{1 z}(\tilde{x}) \hat{x}(x(k, l))
\end{aligned}
$$

where

$$
\begin{aligned}
-Q_{1 z}(\tilde{x}) & =\left(\tilde{A}_{z}(\tilde{x})+\tilde{B}_{z}(\tilde{x}) F_{z}(\tilde{x})\right)^{T} P_{z}\left(\tilde{x}^{+}\right) \\
& \times\left(\tilde{A}_{z}(\tilde{x})+\tilde{B}_{z}(\tilde{x}) F_{z}(\tilde{x})\right)-P_{z}(\tilde{x})
\end{aligned}
$$

and

$$
\begin{aligned}
\tilde{A}_{z}(\tilde{x}) & =T\left(\tilde{x}^{+}\right) A_{z}(\tilde{x}), \tilde{B}_{z}(\tilde{x})=T\left(\tilde{x}^{+}\right) B_{z}(\tilde{x}) \\
T\left(\tilde{x}^{+}\right) & =\left[\begin{array}{cc}
T_{1}\left(\tilde{x}^{+}\right) & 0 \\
0 & T_{2}\left(\tilde{x}^{+}\right)
\end{array}\right]=\left[\begin{array}{cc}
T_{1}(x(k+1, l) & 0 \\
0 & T_{2}(x(k, l+1))
\end{array}\right]
\end{aligned}
$$

Note that $Q_{1 z}(\tilde{x})$ are positive definite symmetric polynomial matrices, and $\lambda_{\min }\left(Q_{1 z}(\tilde{x})\right)>0$, so

$$
\hat{x}^{T}(x(k, l)) Q_{1 z}(\tilde{x}) \hat{x}(x(k, l)) \geq \lambda_{\min }\left(Q_{1 z}(\tilde{x})\right)\|\hat{x}(x(k, l))\|_{2}^{2}
$$

Thus, $\Delta V(x(k, l))$ is bounded as follows:

$$
\begin{aligned}
& \Delta V(x(k, l)) \leq-\lambda_{\min }\left(Q_{1 z}(\tilde{x})\right)\|\hat{x}(x(k, l))\|_{2}^{2}, \forall\|\hat{x}(x(k, l))\|_{2}<\eta_{(N)} \\
& \begin{aligned}
\Delta V(x(k, l)) \text { is negative if }-Q_{1 z}(\tilde{x})<0 \\
-Q_{1 z}(\tilde{x})=\left[( \tilde { A } _ { z } ( \tilde { x } ) + \tilde { B } _ { z } ( \tilde { x } ) F _ { z } ( \tilde { x } ) ) ^ { T } P _ { z } ( \tilde { x } ^ { + } ) \left(\tilde{A}_{z}(\tilde{x})\right.\right. \\
\left.\left.+\tilde{B}_{z}(\tilde{x}) F_{z}(\tilde{x})\right)-P_{z}(\tilde{x})\right]<0
\end{aligned}
\end{aligned}
$$

Pre- and post-multiplying both sides of (17) by $P_{z}^{-1}(\tilde{x})$, we obtain (18) where $P_{z}^{-1}(\tilde{x})=X_{z}(\tilde{x})$ and $K_{z}(\tilde{x})=F_{z}(\tilde{x}) X_{z}(\tilde{x})$, $i=1, \ldots, r$ :

$$
\begin{aligned}
& {\left[X _ { z } ( \tilde { x } ) ( \tilde { A } _ { z } ( \tilde { x } ) + \tilde { B } _ { z } ( \tilde { x } ) F _ { z } ( \tilde { x } ) ) ^ { T } X _ { z } ^ { - 1 } ( \tilde { x } ^ { + } ) \left(\tilde{A}_{z}(\tilde{x})\right.\right.} \\
& \left.\left.+\tilde{B}_{z}(\tilde{x}) F_{z}(\tilde{x})\right) X_{z}(\tilde{x})-X_{z}(\tilde{x})\right]<0
\end{aligned}
$$

applying the Schur complement, we then have

$$
\Omega_{z}^{m n}(\tilde{x})=\left[\begin{array}{cc}
X_{z}(\tilde{x}) & \left(\tilde{A}_{z}(\tilde{x}) X_{z}(\tilde{x})+\tilde{B}_{z}(\tilde{x}) K_{z}(\tilde{x})\right)^{T} \\
* & X_{z}\left(\tilde{x}^{+}\right)
\end{array}\right]>0
$$

where

$$
\begin{gathered}
\Omega_{z}^{m n}(\tilde{x})=\sum_{m=1}^{r} \sum_{n=1}^{r} \sum_{i=1}^{r} \sum_{j=1}^{r} h_{m}(z(k+1, l)) h_{n}(z(k, l+1)) h_{i} h_{j} \Omega_{i j}^{m n}(\tilde{x}) \\
X_{z}(\tilde{x})=\left[\begin{array}{cc}
X_{1}^{z}(\tilde{x}) & 0 \\
0 & X_{2}^{z}(\tilde{x})
\end{array}\right], X_{z}\left(\tilde{x}^{+}\right)=\left[\begin{array}{cc}
X_{1}^{z}\left(\tilde{x}^{+}\right) & 0 \\
0 & X_{2}^{z}\left(\tilde{x}^{+}\right)
\end{array}\right] \\
X_{1}^{z}\left(\tilde{x}^{+}\right)=\sum_{m=1}^{r} h_{m}(z(k+1, l)) X_{1}^{m}(x(k+1, l))
\end{gathered}
$$

$$
X_{2}^{z}\left(\tilde{x}^{+}\right)=\sum_{n=1}^{r} h_{n}(z(k, l+1)) X_{2}^{n}(x(k, l+1))
$$

and $\Omega_{i j}^{m n}(\tilde{x})$ are defined in (14). 
3.2 Stabilization Conditions for Polynomial System using Non-PDC Control

In order to obtain more relaxed stabilization conditions for polynomial discrete 2-D T-S systems, a Non-PDC control law is used, of the following form [28]:

$u(k, l)=K_{z}(\tilde{x}) Y_{z}^{-1}(\tilde{x}) \hat{x}(x(k, l))$

where $K_{z}(\tilde{x})$ and $Y_{z}(\tilde{x})$ are polynomial matrices of appropriate dimensions to be determined

$K_{z}(\tilde{x})=\left[K_{1}^{z}(\tilde{x}) K_{2}^{z}(\tilde{x})\right], Y_{z}(\tilde{x})=\left[\begin{array}{cc}Y_{1}^{z}(\tilde{x}) & 0 \\ 0 & Y_{2}^{z}(\tilde{x})\end{array}\right]$

the closed-loop system of (4) and (20) is then as follows:

$x^{+}(k, l)=\left(A_{z}(\tilde{x})+B_{z}(\tilde{x}) K_{z}(\tilde{x}) Y_{z}^{-1}(\tilde{x})\right) \hat{x}(x(k, l))$

Theorem 2 The 2-D system (21) is asymptotically stable if there exist symmetric polynomial matrices $P_{1}^{i}(\tilde{x}) \in \mathbb{R}^{n_{1} \times n_{1}}$, $P_{2}^{i}(\tilde{x}) \in \mathbb{R}^{n_{2} \times n_{2}}$, and polynomial matrices $K_{1}^{i}(\tilde{x}) \in \mathbb{R}^{m_{1} \times n_{1}}$, $K_{2}^{i}(\tilde{x}) \in \mathbb{R}^{m_{2} \times n_{2}}, Y_{1}^{i}(\tilde{x}) \in \mathbb{R}^{n_{1} \times n_{1}}, Y_{2}^{i}(\tilde{x}) \in \mathbb{R}^{n_{2} \times n_{2}}, Z_{i i}^{m n}(\tilde{x})$, $Z_{i j}^{m n}(\tilde{x})=\left(Z_{j i}^{m n}\right)^{T}(\tilde{x})$ such that (22), (23), (24), (25) and (26) are satisfied, where $\varepsilon_{1}^{i}(\tilde{x}), \varepsilon_{2}^{i}(\tilde{x}), \varepsilon_{1}^{i j}(\tilde{x})$ and $\varepsilon_{2}^{i j}(\tilde{x})$ are non negative polynomials such that $\varepsilon_{1}^{i}(\tilde{x})>0, \varepsilon_{2}^{i}(\tilde{x})>0$ for $(\tilde{x} \neq$ $0)$ and $\varepsilon_{1}^{i j}(\tilde{x}) \geq 0, \varepsilon_{2}^{i j}(\tilde{x}) \geq 0$ for all $\tilde{x}$.

$v_{1}^{T}\left(P_{1}^{i}(\tilde{x})-\varepsilon_{1}^{i}(\tilde{x}) I\right) v_{1} \quad$ is $\operatorname{SOS}$

$v_{1}^{T}\left(P_{2}^{i}(\tilde{x})-\varepsilon_{2}^{i}(\tilde{x}) I\right) v_{1} \quad$ is SOS

$v_{2}^{T}\left(T_{i i}^{m n}(\tilde{x})-Z_{i i}^{m n}(\tilde{x})\right) v_{2}$ is $\operatorname{SOS} i, m, n=1, \ldots, r$

$v_{2}^{T}\left(T_{i j}^{m n}(\tilde{x})+T_{j i}^{m n}(\tilde{x})-Z_{i j}^{m n}(\tilde{x})-Z_{j i}^{m n}(\tilde{x})\right) v_{2} \quad$ is SOS

$i<j, \quad i, j, m, n=1, \ldots, r$.

$\left[\begin{array}{cccc}Z_{11}^{m n}(\tilde{x}) & Z_{12}^{m n}(\tilde{x}) & \ldots & Z_{1 r}^{m n}(\tilde{x}) \\ Z_{21}^{m n}(\tilde{x}) & Z_{22}^{m n}(\tilde{x}) & \ldots & Z_{2 r}^{m n}(\tilde{x}) \\ \vdots & \vdots & \ddots & \vdots \\ Z_{r 1}^{m n}(\tilde{x}) & Z_{r 2}^{m n}(\tilde{x}) & \ldots & Z_{r r}^{m n}(\tilde{x})\end{array}\right]>0$

$m, n=1, \ldots, r$.

with $v_{1}$ and $v_{2}$ vectors that are independent of $x(k, l)$.

$\begin{aligned} T_{i j}^{m n}(\tilde{x}) & =\left[\begin{array}{cccc}T_{i j 11}(\tilde{x}) & * & * & * \\ 0 & T_{i j 22}(\tilde{x}) & * & * \\ T_{i j 31}(\tilde{x}) & T_{i j 32}(\tilde{x}) & T_{i j 33}^{m}(\tilde{x}) & * \\ T_{i j 41}(\tilde{x}) & T_{i j 42}(\tilde{x}) & 0 & T_{i j 44}^{n}(\tilde{x})\end{array}\right] \\ & >0\end{aligned}$

$$
\begin{aligned}
T_{i j 11}(\tilde{x})= & P_{1}^{j}(\tilde{x})-\varepsilon_{1}^{i}(\tilde{x}) I \\
T_{i j 22}(\tilde{x})= & P_{2}^{j}(\tilde{x})-\varepsilon_{2}^{i}(\tilde{x}) I \\
T_{i j 31}(\tilde{x})= & T_{1}\left(\tilde{x}^{+}\right) A_{11}^{i}(\tilde{x}) Y_{1}^{j}(\tilde{x})+T_{1}\left(\tilde{x}^{+}\right) B_{1}^{i}(\tilde{x}) K_{1}^{j}(\tilde{x}) \\
T_{i j 32}(\tilde{x})= & T_{1}\left(\tilde{x}^{+}\right) A_{12}^{i}(\tilde{x}) Y_{2}^{j}(\tilde{x})+T_{1}\left(\tilde{x}^{+}\right) B_{1}^{i}(\tilde{x}) K_{2}^{j}(\tilde{x}) \\
T_{i j 33}^{m}(\tilde{x})= & Y_{1}^{m}(x(k+1, l))+Y_{1}^{m T}(x(k+1, l)) \\
& -P_{1}^{m}(x(k+1, l))-\varepsilon_{1}^{i j}(\tilde{x}) I \\
T_{i j 41}(\tilde{x})= & T_{2}\left(\tilde{x}^{+}\right) A_{21}^{i}(\tilde{x}) Y_{1}^{j}(\tilde{x})+T_{2}\left(\tilde{x}^{+}\right) B_{2}^{i}(\tilde{x}) K_{1}^{j}(\tilde{x}) \\
T_{i j 42}(\tilde{x})= & T_{2}\left(\tilde{x}^{+}\right) A_{22}^{i}(\tilde{x}) Y_{2}^{j}(\tilde{x})+T_{2}\left(\tilde{x}^{+}\right) B_{2}^{i}(\tilde{x}) K_{2}^{j}(\tilde{x}) \\
T_{i j 44}^{n}(\tilde{x})= & Y_{2}^{n}(x(k, l+1))+Y_{2}^{n T}(x(k, l+1)) \\
& -P_{2}^{n}\left(x(k, l+1)-\varepsilon_{2}^{i j}(\tilde{x}) I\right.
\end{aligned}
$$

Proof Consider the following polynomial Lyapunov function [28]:

$V(x(k, l))=\hat{x}^{T}(x(k, l)) Y_{z}^{-T}(\tilde{x}) P_{z}(\tilde{x}) Y_{z}^{-1}(\tilde{x}) \hat{x}(x(k, l))$

Then,

$\hat{x}^{T}(x(k, l)) v_{1 \min } Y_{z}^{-T}(\tilde{x}) Y_{z}^{-1}(\tilde{x}) \hat{x}(x(k, l)) \leq V(x(k, l))$

$$
\leq \hat{x}^{T}(x(k, l)) v_{1 \max } Y_{z}^{-T}(\tilde{x}) Y_{z}^{-1}(\tilde{x}) \hat{x}(x(k, l))
$$

where

$v_{1 \min }=\lambda_{\min _{z}}\left(P_{z}(\tilde{x})\right), \quad v_{1 \max }=\lambda_{\max _{z}}\left(P_{z}(\tilde{x})\right)$

As $\left(Y_{z}^{-T}(\tilde{x}) Y_{z}^{-1}(\tilde{x})\right)=Y_{z}(\tilde{x}) Y_{z}^{T}(\tilde{x})$ with

$v_{2 \min }=\lambda_{\min _{z}}\left(Y_{z}(\tilde{x}) Y_{z}^{T}(\tilde{x})\right), \quad v_{2 \max }=\lambda_{\max _{z}}\left(Y_{z}(\tilde{x}) Y_{z}^{T}(\tilde{x})\right)$

(29) becomes

$v_{1 \min } v_{2 \max }^{-1}\|\hat{x}(x(k, l))\|^{2} \leq V(x(k, l)) \leq v_{1 \max } v_{2 \min }^{-1}\|\hat{x}(x(k, l))\|^{2}$

which ensures that $V(x(k, l))$ is a polynomial Lyapunov function.

Then, the variation of (28) is given by

$$
\begin{aligned}
\Delta V(x(k, l)) & =\hat{x}^{T}(x(k, l))\left[\left(\tilde{A}_{z}(\tilde{x})+\tilde{B}_{z}(\tilde{x}) K_{z}(\tilde{x})\right.\right. \\
& \left.\times Y_{z}^{-1}(\tilde{x})\right)^{T} Y_{z}^{-T}\left(\tilde{x}^{+}\right) P_{z}\left(\tilde{x}^{+}\right) Y_{z}^{-1}\left(\tilde{x}^{+}\right)\left(\tilde{A}_{z}(\tilde{x})\right. \\
& \left.+\tilde{B}_{z}(\tilde{x}) K_{z}(\tilde{x}) Y_{z}^{-1}(\tilde{x})\right) \\
& \left.-Y_{z}^{-T}(\tilde{x}) P_{z}(\tilde{x}) Y_{z}^{-1}(\tilde{x})\right] \hat{x}(x(k, l)) \\
& =-\hat{x}^{T}(x(k, l)) Q_{2 z}(\tilde{x}) \hat{x}(x(k, l))
\end{aligned}
$$

where

$$
\begin{aligned}
-Q_{2 z}(\tilde{x}) & =\left[\left(\tilde{A}_{z}(\tilde{x})+\tilde{B}_{z}(\tilde{x}) K_{z}(\tilde{x})\right.\right. \\
& \left.\times Y_{z}^{-1}(\tilde{x})\right)^{T} Y_{z}^{-T}\left(\tilde{x}^{+}\right) P_{z}\left(\tilde{x}^{+}\right) Y_{z}^{-1}\left(\tilde{x}^{+}\right)\left(\tilde{A}_{z}(\tilde{x})\right. \\
& \left.+\tilde{B}_{z}(\tilde{x}) K_{z}(\tilde{x}) Y_{z}^{-1}(\tilde{x})\right) \\
& \left.-Y_{z}^{-T}(\tilde{x}) P_{z}(\tilde{x}) Y_{z}^{-1}(\tilde{x})\right]
\end{aligned}
$$


$Y^{z}\left(\tilde{x}^{+}\right)=\left[\begin{array}{cc}Y_{1}^{z}\left(\tilde{x}^{+}\right) & 0 \\ 0 & Y_{2}^{z}\left(\tilde{x}^{+}\right)\end{array}\right], P^{z}\left(\tilde{x}^{+}\right)=\left[\begin{array}{cc}P_{1}^{z}\left(\tilde{x}^{+}\right) & 0 \\ 0 & P_{2}^{z}\left(\tilde{x}^{+}\right)\end{array}\right]$

$Y_{1}^{z}\left(\tilde{x}^{+}\right)=\sum_{m=1}^{r} h_{m}(z(k+1, l)) Y_{1}^{m}(x(k+1, l))$

$Y_{2}^{z}\left(\tilde{x}^{+}\right)=\sum_{n=1}^{r} h_{n}(z(k, l+1)) Y_{2}^{n}(x(k, l+1))$

$P_{1}^{z}\left(\tilde{x}^{+}\right)=\sum_{m=1}^{r} h_{m}(z(k+1, l)) P_{1}^{m}(x(k+1, l))$

$P_{2}^{z}\left(\tilde{x}^{+}\right)=\sum_{n=1}^{r} h_{n}(z(k, l+1)) P_{2}^{n}(x(k, l+1))$

and $Q_{2 z}(\tilde{x})$ are symmetric polynomial matrices, positive definite.

$\hat{x}^{T}(x(k, l)) Q_{2 z}(\tilde{x}) \hat{x}(x(k, l)) \geq \lambda_{\min }\left(Q_{2 z}(\tilde{x})\right)\|\hat{x}(x(k, l))\|_{2}^{2}$

Thus, $\Delta V(x(k, l))$ is bounded as follows:

$$
\begin{gathered}
\Delta V(x(k, l)) \leq-\lambda_{\min }\left(Q_{2 z}(\tilde{x})\right)\|\hat{x}(x(k, l))\|_{2}^{2}, \\
\forall\|\hat{x}(x(k, l))\|_{2}<\eta_{(N)}
\end{gathered}
$$

Note that in the 2-D system the information is propagated along two independent directions; hence, $h_{i}(z)(k+$ $1, l))$ and $h_{j}(z(k, l+1))$ are two different membership functions.

Multiplying the right side of (31) by $Y_{z}(\tilde{x})$, and the left side by $Y_{z}^{T}(\tilde{x})$, we have:

$$
\begin{aligned}
& \left(Y_{z}^{T}(\tilde{x}) \tilde{A}_{z}^{T}(\tilde{x})+K_{z}^{T}(\tilde{x}) \tilde{B}_{z}^{T}(\tilde{x})\right) Y_{z}^{-T}\left(\tilde{x}^{+}\right) P_{z}\left(\tilde{x}^{+}\right) Y_{z}^{-1}\left(\tilde{x}^{+}\right) \\
& \times\left(\tilde{A}_{z}(\tilde{x}) Y_{z}(\tilde{x})+\tilde{B}_{z}(\tilde{x}) K_{z}(\tilde{x})\right)-P_{z}(\tilde{x})<0 .
\end{aligned}
$$

By using Lemma 1 , where $A=Y_{z}^{-1}\left(\tilde{x}^{+}\right)\left(\tilde{A}_{z}(\tilde{x}) Y_{z}(\tilde{x})+\tilde{B}_{z}(\tilde{x}) K_{z}(\tilde{x})\right)$,

(32) can be expressed as

$T_{z}(\tilde{x})=\left[\begin{array}{cc}P_{z}(\tilde{x}) & * \\ \Upsilon_{21} & \Upsilon_{22}\end{array}\right]>0$

where

$$
\begin{aligned}
\Upsilon_{21}= & \tilde{A}_{z}(\tilde{x}) Y_{z}(\tilde{x})+\tilde{B}_{z}(\tilde{x}) K_{z}(\tilde{x}) \\
\Upsilon_{22}= & Y_{z}\left(\tilde{x}^{+}\right)+Y_{z}^{T}\left(\tilde{x}^{+}\right)-P_{z}\left(\tilde{x}^{+}\right) \\
T_{z}(\tilde{x}) & =\sum_{m=1}^{r} \sum_{n=1}^{r} h_{m}(z(k+1, l)) h_{n}(z(k, l+1)) \\
& \times\left(\sum_{i=1}^{r} h_{i}^{2} T_{i i}^{m n}(\tilde{x})+\sum_{i=1}^{r-1} \sum_{j>i} h_{i} h_{j}\left(T_{i j}^{m n}(\tilde{x})+T_{j i}^{m n}(\tilde{x})\right)\right)
\end{aligned}
$$

where $T_{i j}^{m n}(\tilde{x})$ are defined in (27)
If the conditions (25) and (26) are satisfied, then the following are also satisfied:

$$
\begin{aligned}
T_{z}(\tilde{x}) & \geq \sum_{m=1}^{r} \sum_{n=1}^{r} h_{m}(z(k+1, l)) h_{n}(z(k, l+1)) \\
& \times\left(\sum_{i=1}^{r} h_{i}^{2} Z_{i i}^{m n}(\tilde{x})+\sum_{i=1}^{r-1} \sum_{j>i} h_{i} h_{j}\left(Z_{i j}^{m n}(\tilde{x})+Z_{j i}^{m n}(\tilde{x})\right)\right) \\
& =\sum_{m=1}^{r} \sum_{n=1}^{r} h_{m}(z(k+1, l)) h_{n}(z(k, l+1)) \theta^{T} Z^{m n}(\tilde{x}) \theta
\end{aligned}
$$

where $\theta^{T}=\left[\begin{array}{llll}h_{1} I & h_{2} I & \ldots & h_{r} I\end{array}\right]$ and

$$
Z^{m n}(\tilde{x})=\left[\begin{array}{cccc}
Z_{11}^{m n}(\tilde{x}) & Z_{12}^{m n}(\tilde{x}) & \ldots & Z_{1 r}^{m n}(\tilde{x}) \\
Z_{21}^{m n}(\tilde{x}) & Z_{22}^{m n}(\tilde{x}) & \ldots & Z_{2 r}^{m n}(\tilde{x}) \\
\vdots & \vdots & \ddots & \vdots \\
Z_{r 1}^{m n}(\tilde{x}) & Z_{r 2}^{m n}(\tilde{x}) & \ldots & Z_{r r}^{m n}(\tilde{x})
\end{array}\right]
$$

Remark 2 The feasibility of the developed SOS condition is influenced by the polynomials $\varepsilon_{i}^{1}(x), \varepsilon_{i}^{2}(x), \varepsilon_{i j}^{1}(x)$ and $\varepsilon_{i j}^{2}(x)$; therefore, in practice the polynomial structure of $\varepsilon_{i}^{1}(x), \varepsilon_{i}^{2}(x)$, $\varepsilon_{i j}^{1}(x)$ and $\varepsilon_{i j}^{2}(x)$ has to be carefully selected.

Remark 3 Following the previous study on polynomial fuzzy models, [24,33], the major drawback of the results is the numerical computational cost. In fact, when the degree $2 d$ increases, the computational complexity increases, and the computational time required increases. Nonetheless, the SOS approach has a clear advantage, as a generalization of the existing approaches to T-S fuzzy system, being more effective in representing nonlinear control systems. In fact, the SOS approach used here is more relaxed than the LMI approach previously used in the literature [32].

\section{Computer simulations}

Example 1 Let us consider the following nonlinear differential equation borrowed from [32]

$$
\begin{aligned}
\frac{\partial^{2} q(x, t)}{\partial x \partial t} & =a_{1} \frac{\partial q(x, t)}{\partial t}+a_{2} \frac{\partial q(x, t)}{\partial x}+a_{0} \sin ^{2}(q(x, t)) \\
& +b f(x, t))
\end{aligned}
$$

with the initial and boundary conditions $q(x, 0)=q_{1}(x)$ and $q(t, 0)=q_{2}(t) . q(x, t)$ is the state function, $a_{0}, a_{1}, a_{2}, b$ are real coefficients, and $f(x, t)$ is the input function. If we define

$$
\begin{aligned}
& x_{c}^{h}(x, t)=\frac{\partial q(x, t)}{\partial t}-a_{2} q(x, t) \\
& x_{c}^{v}(x, t)=q(x, t)
\end{aligned}
$$


then we obtain the following 2-D model:

$$
\begin{aligned}
{\left[\begin{array}{l}
\frac{\partial x_{c}^{h}(x, t)}{\partial x} \\
\frac{\partial x_{c}^{v}(x, t)}{\partial t}
\end{array}\right] } & =\left[\begin{array}{cc}
a_{1} & a_{1} a_{2}+a_{0} \sin ^{2}\left(x_{c}^{v}(x, t)\right) \\
1 & a_{2}
\end{array}\right] \\
& \times\left[\begin{array}{l}
x_{c}^{h}(x, t) \\
x_{c}^{v}(x, t)
\end{array}\right]+\left[\begin{array}{l}
b \\
0
\end{array}\right] u_{c}(x, t)
\end{aligned}
$$

with boundary conditions

$x_{c}^{h}(0, t)=\dot{q}_{2}(t)-a_{2} q_{2}(t)$

$x_{c}^{v}(x, t)=q_{1}(x)$

Consider the two following rules obtained for $\sin ^{2}\left(x_{c}^{v}(x, t)\right)$ :

$$
\text { IF } \sin ^{2}\left(x_{c}^{v}(x, t)\right) \text { is about 0, THEN }
$$

$\left[\begin{array}{l}\frac{\partial x_{c}^{h}(x, t)}{\partial t} \\ \frac{\partial x_{c}^{v}(x, t)}{\partial t}\end{array}\right]=A_{1}^{c}\left[\begin{array}{l}x_{c}^{h}(x, t) \\ x_{c}^{v}(x, t)\end{array}\right]+B_{1}^{c} u_{c}(x, t)$

IF $\sin ^{2}\left(x_{c}^{v}(x, t)\right)$ is about $\mp 1$, THEN

$\left[\begin{array}{l}\frac{\partial x_{c}^{h}(x, t)}{\partial t} \\ \frac{\partial x_{c}^{v}(x, t)}{\partial t}\end{array}\right]=A_{2}^{c}\left[\begin{array}{l}x_{c}^{h}(x, t) \\ x_{c}^{v}(x, t)\end{array}\right]+B_{2}^{c} u_{c}(x, t)$

with

$A_{1}^{c}=\left[\begin{array}{cc}a_{1} & a_{1} a_{2} \\ 1 & a_{2}\end{array}\right], B_{1}^{c}=\left[\begin{array}{l}b \\ 0\end{array}\right]$

$A_{2}^{c}=\left[\begin{array}{cc}a_{1} & a_{1} a_{2}+a_{0} \\ 1 & a_{2}\end{array}\right], B_{2}^{c}=B_{1}^{c}$

The membership functions are given by:

$h_{1}(x, t)=1-\sin ^{2}\left(x_{c}^{v}(x, t)\right), h_{2}(x, t)=\sin ^{2}\left(x_{c}^{v}(x, t)\right)$

For control purpose the 2-D T-S system is discretized with sampling intervals $T_{1}$ and $T_{2}$ corresponding to variables $x$ and $t$, respectively, with the system parameters given in [32,29] and [34], obtaining the following system:

IF $\sin ^{2}\left(x^{v}(k, l)\right)$ is about 0, THEN

$\left[\begin{array}{l}x^{h}(k+1, l) \\ x^{v}(k, l+1)\end{array}\right]=A_{1}\left[\begin{array}{l}x^{h}(k, l) \\ x^{v}(k, l)\end{array}\right]+B_{1} u(k, l)$

IF $\sin ^{2}\left(x^{v}(k, l)\right)$ is about $\mp 1$, THEN

$\left[\begin{array}{l}x^{h}(k+1, l) \\ x^{v}(k, l+1)\end{array}\right]=A_{2}\left[\begin{array}{l}x^{h}(k, l) \\ x^{v}(k, l)\end{array}\right]+B_{2} u(k, l)$

$A_{1}=\left[\begin{array}{cc}1+a_{1} T_{1} & a_{1} a_{2} T_{1} \\ T_{2} & 1+a_{2} T_{2}\end{array}\right], B_{1}=\left[\begin{array}{c}b T_{1} \\ 0\end{array}\right]$

$A_{2}=\left[\begin{array}{cc}1+a_{1} T_{1} & \left(a_{1} a_{2}+a_{0}\right) T_{1} \\ T_{2} & 1+a_{2} T_{2}\end{array}\right], B_{2}=B_{1}$

The membership functions then become: $h_{1}(k, l)=1-$ $\sin ^{2}\left(x^{v}(k, l)\right), h_{2}(k, l)=\sin ^{2}\left(x^{v}(k, l)\right)$. We can refer to [40]
Table 1 Feasibility intervals for $a_{2}$

\begin{tabular}{cc}
\hline Methods & Feasibility intervals \\
\hline Usual PDC [32] & {$[-1.990,-0.512]$} \\
Theorem 1 of [32] & {$[-2.012,-0.494]$} \\
Corollary 2 [32] & {$[-2.292,-0.212]$} \\
Theorem 2 non-PDC of [32] & {$[-2.492,-0.014]$} \\
Theorem 1 PDC of [28] & {$[-2.499,-0.018]$} \\
Theorem 2 non-PDC of [28] & {$[-2.65,-0.01]$} \\
Theorem 1 of [29] with $g=2$ & {$[-2.502,-0.013]$} \\
Theorem 2 of [29] with $g=d=2$ & {$[-2.561,-0.012]$} \\
Theorem 3 of [29] with $g=d=2$ & {$[-2.566,-0.011]$} \\
Theorem 1 PDC with $2 d=2$ & {$[-2.525,-0.001]$} \\
Theorem 2 non-PDC with $2 d=2$ & {$[-2.743,-0.001]$} \\
\hline
\end{tabular}

for more discussions about the discretization of the continuoustime T-S fuzzy model, see also the papers dealing with the delta operator, like [41]. For the numerical simulation we consider the following parameters: $a_{1}=-3, a_{0}=-2, b=$ $-1, T_{1}=0.5, T_{2}=0.8$.

The feasible intervals for $a_{2}$ are shown in Table 1 for the proposed technique, and compared with those in the literature. From Table 1, we can observe that the intervals obtained by our proposed Theorems 1 and 2 are larger than the results given by [32], [29] and [28]. Fixing $a_{2}=-2.4$, and then solving (22) to (26) with the SOSTOOLS solver gives:

$$
K_{1}(\tilde{x})=\left[K_{1}^{1}(\tilde{x}) K_{2}^{1}(\tilde{x})\right], K_{2}(\tilde{x})=\left[K_{1}^{2}(\tilde{x}) K_{2}^{2}(\tilde{x})\right]
$$

$$
Y_{1}(\tilde{x})=\left[\begin{array}{cc}
Y_{1}^{1}(\tilde{x}) & 0 \\
0 & Y_{2}^{1}(\tilde{x})
\end{array}\right], Y_{2}(\tilde{x})=\left[\begin{array}{cc}
Y_{1}^{2}(\tilde{x}) & 0 \\
0 & Y_{2}^{2}(\tilde{x})
\end{array}\right]
$$

$K_{1}^{1}(\tilde{x})=-7.5365 .10^{-6} x^{h}(k, l)^{2}-435.36 x^{v}(k, l)^{2}$

$K_{2}^{1}(\tilde{x})=0.000070999 x^{h}(k, l)^{2}+14012.0 x^{v}(k, l)^{2}$

$K_{1}^{2}(\tilde{x})=-7.2643 .10^{-6} x^{h}(k, l)^{2}-433.25 x^{v}(k, l)^{2}$

$K_{2}^{2}(\tilde{x})=0.000051495 x^{h}(k, l)^{2}+10150.0 x^{v}(k, l)^{2}$

$Y_{1}^{1}(\tilde{x})=7.0585 .10^{-6} x^{h}(k, l)^{2}+427.83 x^{v}(k, l)^{2}$

$Y_{2}^{1}(\tilde{x})=0.000010406 x^{h}(k, l)^{2}+1945.4 x^{v}(k, l)^{2}$

$Y_{1}^{2}(\tilde{x})=7.1543 .10^{-6} x^{h}(k, l)^{2}+428.39 x^{v}(k, l)^{2}$

$Y_{2}^{2}(\tilde{x})=0.000010334 x^{h}(k, l)^{2}+1951.3 x^{v}(k, l)^{2}$

with the boundary conditions

$x^{h}(0, l)=0.2, \quad 0 \leq l \leq 30$

$x^{v}(k, 0)=0.3, \quad 0 \leq k \leq 30$

$x^{h}(0, l)=0.05, \quad x^{v}(k, 0)=0.1, \quad i, j>30$ 


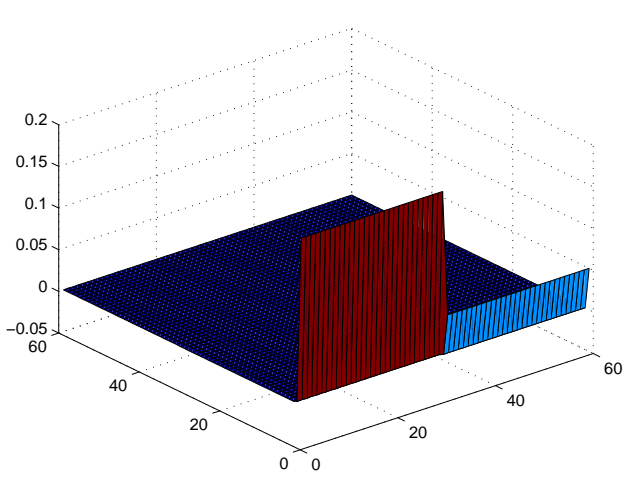

Fig. 1 Horizontal states $x^{h}(k, l)$ for $a_{2}=-2.4$ using the proposed controller.

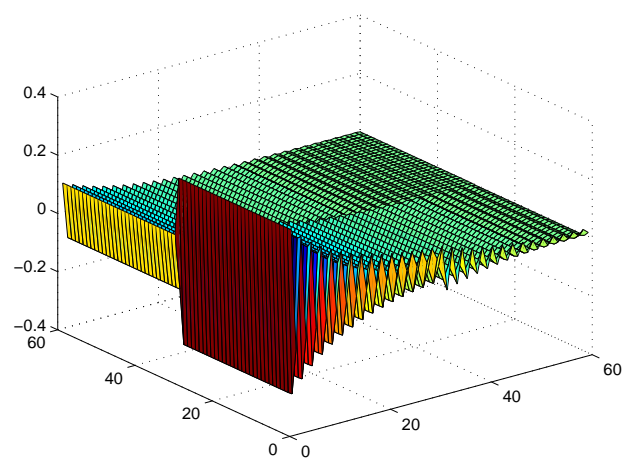

Fig. 2 Vertical states $x^{v}(k, l)$ for $a_{2}=-2.4$ using the proposed controller.

Figures 1 and 2 show the evolutions of the two states $x^{h}(k, l)$ and $x^{v}(k, l)$, respectively: it is clear that the polynomial discrete 2-D T-S system (4) with the polynomial controller (20) is asymptotically stable.

Remark 4 The same system can be studied with the saturation. In fact, 2-D systems with saturation has been already studied in [39] (FM T-S system) where the sinusoid was used in the example. The 2-D saturated systems for Roesser model was also solved in [38], where the saturation function used is the standard symmetric one. Note that we have already dealt with the problem of stabilization of nonlinear discrete-time T-S fuzzy systems with actuator saturation in [37] for (1-D), where the sinusoid was used in the example, and we will consider the discrete 2-D T-S problem as future work.

For a polynomial $X(\tilde{x}) \in \mathbb{R}^{n_{d} \times n_{d}}$, the complexity of computing the SOS decomposition, depends on two factors: the number of variables and the degree of the polynomial $(2 d+$ 1) $n_{d}^{2}$.

The number of variable (N.V) and the number of (LMIs / SOS) are shown in Table 2 for the example. Notice that with this technique lies when the number of variables or the de- gree of the polynomial are increased, the conservatism of the result decreases, but the computational complexity increases.

Example 2 Consider the following discrete-time nonlinear plant represented by the polynomial 2-D T-S fuzzy Roesser model with the following two rules:

$$
\begin{aligned}
& A_{1}=\left[\begin{array}{cc}
0.25 & 0.675 \\
0.75 x^{h}(k, l) & 0.325
\end{array}\right], B_{1}=\left[\begin{array}{c}
-0.36 x^{v}(k, l) \\
0
\end{array}\right] \\
& A_{2}=\left[\begin{array}{cc}
0.25 & 0.105 \\
0.75 x^{h}(k, l) & 0.325
\end{array}\right], B_{2}=B_{1}
\end{aligned}
$$

The membership functions are given as: $h_{1}(k, l)=1-$ $\sin ^{2}\left(x^{v}(k, l)\right), h_{2}(k, l)=\sin ^{2}\left(x^{v}(k, l)\right)$.

By solving the SOS design condition in Theorems 2, the asymptotical stability for the above 2-D polynomial fuzzy system is ensured. The corresponding controller gain matrices are given by.

$$
\begin{gathered}
K_{1}(\tilde{x})=\left[K_{1}^{1}(\tilde{x}) K_{2}^{1}(\tilde{x})\right], K_{2}(\tilde{x})=\left[K_{1}^{2}(\tilde{x}) K_{2}^{2}(\tilde{x})\right] \\
Y_{1}(\tilde{x})=\left[\begin{array}{cc}
Y_{1}^{1}(\tilde{x}) & 0 \\
0 & Y_{2}^{1}(\tilde{x})
\end{array}\right], Y_{2}(\tilde{x})=\left[\begin{array}{cc}
Y_{1}^{2}(\tilde{x}) & 0 \\
0 & Y_{2}^{2}(\tilde{x})
\end{array}\right]
\end{gathered}
$$

$K_{1}^{1}(\tilde{x})=0.45443 x^{v}(k, l)+3.4487 .10^{-18}$

$K_{2}^{1}(\tilde{x})=1627.9 x^{v}(k, l)-1.0845 .10^{-15}$

$K_{1}^{2}(\tilde{x})=0.45512 x^{v}(k, l)+1.4207 .10^{-17}$

$K_{2}^{2}(\tilde{x})=253.6 x^{v}(k, l)-1.4194 .10^{-17}$

$Y_{1}^{1}(\tilde{x})=6.0479 .10^{-6} x^{h}(k, l)^{2}+6.6041 x^{v}(k, l)^{2}$

$Y_{2}^{1}(\tilde{x})=0.0040157 x^{h}(k, l)^{2}+8681.2 x^{v}(k, l)^{2}$

$Y_{1}^{2}(\tilde{x})=3.9496 .10^{-6} x^{h}(k, l)^{2}+6.609 x^{v}(k, l)^{2}$

$Y_{2}^{2}(\tilde{x})=0.0037871 x^{h}(k, l)^{2}+8693.1 x^{v}(k, l)^{2}$

with the boundary conditions

$$
\begin{aligned}
& x^{h}(0, l)=0.2, \quad 0 \leq l \leq 30 \\
& x^{v}(k, 0)=0.3, \quad 0 \leq k \leq 30 \\
& x^{h}(0, l)=0.05, \quad x^{v}(k, 0)=0.1, \quad i, j>30
\end{aligned}
$$

Figs. 3-4 show the state trajectory of the system state variables $x^{h}(k, l)$ and $x^{v}(k, l)$, respectively. The simulation results in Figs. 3-4, show that the polynomial discrete 2-D T$S$ system is asymptotically stable. Hence, the effectiveness of the proposed approach has been illustrated in the numerical example. 
Table 2 Number of variables and number of (LMIs/ SOS constraints), where $n_{1}=n_{2}=n_{d}$ and $m_{1}=m_{2}=m_{d}$

\begin{tabular}{|c|c|c|}
\hline Methods & N.V & Number of (LMIs/ SOS constraints) \\
\hline Theorem 1 PDC in [32] & $n_{d}\left(n_{d}+1\right)+2 m_{d} n_{d} r+16 n_{d}^{2} r+n_{d}\left(4 n_{d}+1\right) r(r+1)$ & $r+\frac{r}{2}(r-1)+1$ \\
\hline Theorem 1 PDC for degre $2 \mathrm{~d}$ & $(2 d+1)\left[n_{d}\left(n_{d}+1\right) r+2 n_{d} m_{d} r\right]$ & $2 r+r^{3}+\frac{r^{3}}{2}(r-1)$ \\
\hline Theorem 2 NON-PDC in [32] & $n_{d}\left(n_{d}+1\right) r+2 m_{d} n_{d} r+2 n_{d}^{2} r+16 n_{d}^{2} r^{3}+n_{d}\left(4 n_{d}+1\right) r^{3}(r+1)$ & $r^{3}+\frac{r^{3}}{2}(r-1)+r^{2}$ \\
\hline Theorem 2 NON-PDC for degre $2 \mathrm{~d}$ & $(2 d+1)\left[n_{d}\left(n_{d}+1\right) r+2 m_{d} n_{d} r+2 n_{d}^{2} r+16 n_{d}^{2} r^{3}+n_{d}\left(4 n_{d}+1\right) r^{3}(r+1)\right]$ & $2 r+r^{3}+\frac{r^{3}}{2}(r-1)+r^{2}$ \\
\hline
\end{tabular}

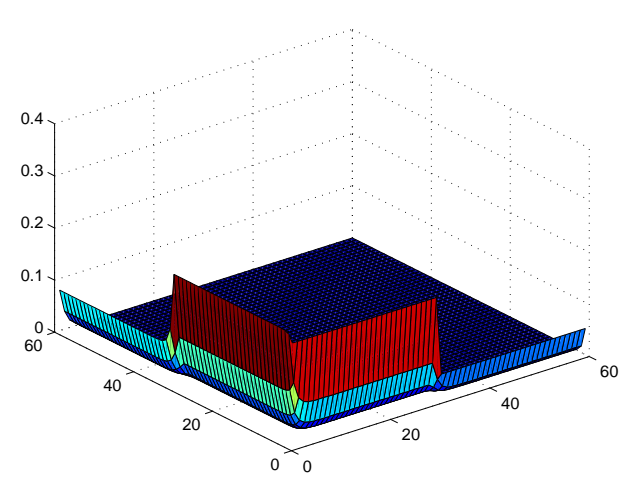

Fig. 3 Trajectory of the 2-D polynomial system state $x^{h}(k, l)$.

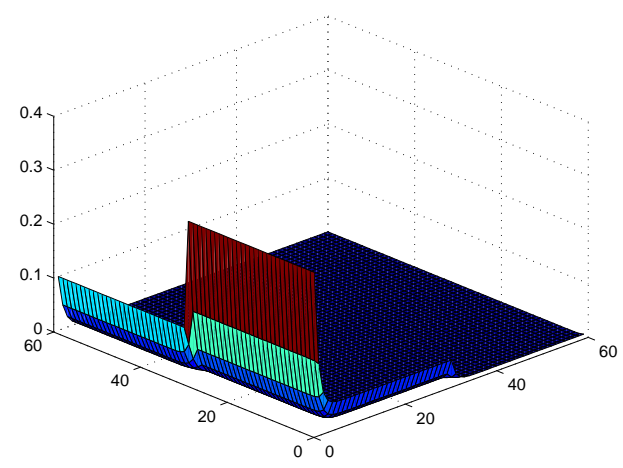

Fig. 4 Trajectory of the 2-D polynomial system state $x^{v}(k, l)$.

\section{Conclusion}

Stabilization of a class of 2-D nonlinear systems has been solved in this paper using a Sum-of-Square approach. More precisely, stabilization of the class of 2-D discrete systems that can be described in terms of Takagi-Sugeno Roessertype polynomial models has been studied using PDC controllers. Firstly, an stabilization condition based on polynomial multiple Lyapunov functions has been derived. Secondly, a non-quadratic stabilization condition was developed using a relaxation technique. These design conditions are formulated in terms of SOS, to make them numerically tractable A numerical example has been provided to show the effectiveness of the proposed results.

\section{Acknowledgment}

This work was partly funded by MiCInn project DPI201454530-R.

\section{References}

1. Rogers, E., and Owens, D. H. (1992). Stability analysis for linear repetitive processes. Springer-Verlag.

2. Sulikowski, B., GalKowski, K., Rogers, E., and Owens, D. H. (2004). Output feedback control of discrete linear repetitive processes. Automatica, 40(12), 2167-2173.

3. Marszalek, W. (1984). Two-dimensional state-space discrete models for hyperbolic partial differential equations. Appl. Math. Model. $8,11-14$

4. Sulikowski, B., Galkowski, K., Rogers, E., and Owens, D. H. (2006). PI control of discrete linear repetitive processes. Automatica, 42(5), 877-880.

5. Owens, D. H., Amann, N., Rogers, E., and French, M. (2000). Analysis of linear iterative learning control schemes-a 2D systems/repetitive processes approach. Multidimensional Systems and Signal Processing, 11(1), 125-177.

6. Du, C., and Xie, L. (2002). $H_{\infty}$ Control and Filtering of TwoDimensional Systems (Vol. 278). Springer Science and Business Media.

7. Boukili, B., Hmamed, A., and Tadeo, F. (2016). Robust $H_{\infty}$ Filtering for 2-D Discrete Roesser Systems. Journal of Control, Automation and Electrical Systems, 27(5), 497-505.

8. El-Kasri, C., Hmamed, A., Tissir, E. H., and Tadeo, F. (2013). Robust $H_{\infty}$ filtering for uncertain two-dimensional continuous systems with time-varying delays. Multidimensional Systems and Signal Processing, 24(4), 685-706.

9. Takagi, T., and Sugeno, M. (1985). Fuzzy identification of systems and its applications to modeling and control. IEEE transactions on Systems, Man, and Cybernetics, (1), 116-132.

10. Xiaodong, L., and Qingling, Z. (2003). New approaches to $H_{\infty}$ controller designs based on fuzzy observers for TS fuzzy systems via LMI. Automatica, 39(9), 1571-1582.

11. Fang, C. H., Liu, Y. S., Kau, S. W., Hong, L., and Lee, C. H. (2006). A new LMI-based approach to relaxed quadratic stabilization of TS fuzzy control systems. IEEE Transactions on Fuzzy Systems, 14(3), 386-397.

12. Wang, H. O., Tanaka, K., and Griffin, M. F. (1996). An approach to fuzzy control of nonlinear systems: Stability and design issues. IEEE Transactions on Fuzzy Systems, 4(1), 14-23.

13. Mozelli, L. A., Palhares, R. M., Souza, F. O., and Mendes, E. M. (2009). Reducing conservativeness in recent stability conditions of TS fuzzy systems. Automatica, 45(6), 1580-1583.

14. Johansson, M., Rantzer, A., and Arzen, K. E. (1999). Piecewise quadratic stability of fuzzy systems. IEEE Transactions on Fuzzy Systems, 7(6), 713-722.

15. Feng, G. (2004). Stability analysis of discrete-time fuzzy dynamic systems based on piecewise Lyapunov functions. IEEE Transactions on Fuzzy Systems, 12(1), 22-28. 
16. Sala, A., and Arino, C. (2007). Relaxed stability and performance conditions for TakagiSugeno fuzzy systems with knowledge on membership function overlap. IEEE Transactions on Systems, Man, and Cybernetics, Part B (Cybernetics), 37(3), 727-732.

17. Chen, J., Xu, S., Zhang, B., Chu, Y., and Zou, Y. (2016). New relaxed stability and stabilization conditions for continuous-time TS fuzzy models. Information Sciences, 329, 447-460.

18. Benzaouia, A., Hmamed, A., \& Tadeo, F. (2016). TwoDimensional Systems. Springer International Publishing: Imprint: Springer,

19. Ding, D., Li, X., Xie, X., and Liu, D. (2011, May). Relaxed stabilization conditions for discrete-time 2-D TS fuzzy systems. In Control and Decision Conference (CCDC), 2011 Chinese (pp. 34593464). IEEE.

20. Boukili, B., Hmamed, A., Benzaouia, A., and El Hajjaji, A. (2014). $H_{\infty}$ filtering of two-dimensional T-S fuzzy systems. Circuits, Systems, and Signal Processing, 33(6), 1737-1761.

21. Boukili, B., Hmamed, A., and Benzaouia, A. (2015). $H_{\infty}$ State Control for 2D Fuzzy FM Systems with Stochastic Perturbation. Circuits, Systems, and Signal Processing, 34(3), 779-796.

22. Prajna, S., Papachristodoulou, A., Seiler, P., and Parrilo, P. A. (2004). SOSTOOLS: Sum of squares optimization toolbox for MATLAB. Users guide, version 2.00

23. Papachristodoulou, A., Anderson, J., Valmorbida, G., Prajna, S., Seiler, P., and Parrilo, P. A. (2013). SOSTOOLS: Sum of squares optimization toolbox for MATLAB Version 3.00.

24. Tanaka, K., Yoshida, H., Ohtake, H., and Wang, H. O. (2007, July). A sum of squares approach to stability analysis of polynomial fuzzy systems. In American Control Conference, 2007. ACC'07 (pp. 40714076). IEEE.

25. Siala, F., Gassara, H., Chaabane, M., and El Hajjaji, A. (2013, December). Stability analysis of polynomial fuzzy systems with timedelay via sum of squares (SOS) approach. In Proc. of 14th International Conference on Sciences and Techniques of Automatic Control and Computer Engineering (STA), (pp. 197-200).

26. Guelton, K., Manamanni, N., Koumba-Emianiwe, D. L., and Chinh, C. D. (2011). SOS stability conditions for nonlinear systems based on a polynomial fuzzy Lyapunov function. IFAC Proceedings Volumes, 44(1), 12777-12782.

27. Shaker, H. R., and Shaker, F. (2014). Lyapunov stability for continuous-time multidimensional nonlinear systems. Nonlinear Dynamics, 75(4), 717-724.

28. Hmamed, A., Chaibi, R., and Tadeo, F. (2016, May). Stabilization of discrete-time 2-D TS Fuzzy system via a sum of squares (SOS) approach. In 5th IEEE International Conference on Systems and Control (ICSC), (pp. 109-114).

29. Xiang-Peng, X. I. E., and ZHANG, H. G. (2010). Convergent stabilization conditions of discrete-time 2-D TS fuzzy systems via improved homogeneous polynomial techniques. Acta Automatica Sinica, 36(9), 1305-1311.

30. de Oliveira, M. C., Bernussou, J., and Geromel, J. C. (1999). A new discrete-time robust stability condition. Systems and Control Letters, 37(4), 261-265.

31. Parrilo, P. A. (2000). Structured semidefinite programs and semialgebraic geometry methods in robustness and optimization (Doctoral dissertation, California Institute of Technology).

32. Xiang-Peng, X., and Zhang, H. G. (2010). Stabilization of discrete-time 2-D TS fuzzy systems based on new relaxed conditions. Acta Automatica Sinica, 36(2), 267-273.

33. Tanaka, K., Ohtake, H., and Wang, H. O. (2008, June). An SOSbased stable control of polynomial discrete fuzzy systems. In IEEE American Control Conference, 2008 pp. 4875-4880.

34. Xie, X. P., Zhang, Z. W., and Hu, S. L. (2015). Control synthesis of roesser type discrete-time 2-dts fuzzy systems via a multi-instant fuzzy state-feedback control scheme. Neurocomputing, 151, 13841391.
35. Silva, L. F., Leite, V. J., Castelan, E. B., and Klug, M. (2014). Local stabilization of time-delay nonlinear discrete-time systems using Takagi-Sugeno models and convex optimization. Mathematical Problems in Engineering, 2014.

36. Dang, Q. V., Vermeiren, L., Dequidt, A., and Dambrine, M. (2017). Robust stabilizing controller design for TakagiSugeno fuzzy descriptor systems under state constraints and actuator saturation. Fuzzy Sets and Systems.

37. Naamane, K., Chaibi, R., Tissir, E. H., and Hmamed, A. (2017, May). Stabilization of discrete-time TS fuzzy systems with saturating actuators. In Advanced Technologies for Signal and Image Processing (ATSIP), 2017 International Conference on (pp. 1-5). IEEE.

38. Hmamed, A., Mesquine, F., Tadeo, F., Benhayoun, M., and Benzaouia, A. (2010). Stabilization of 2D saturated systems by state feedback control. Multidimensional Systems and Signal Processing, 21(3), 277-292.

39. Gao, J., and Wang, W. (2014, July). $H_{\infty}$ control For 2-D fuzzy FM II system with actuator saturation. In Proc. of the 33rd Chinese Control Conference (CCC), pp. 4435-4439.

40. Li, Z., Park, J. B., \& Joo, Y. H. (2001). Chaotifying continuoustime TS fuzzy systems via discretization. IEEE Transactions on Circuits and Systems I: Fundamental Theory and Applications, 48(10), 1237-1243.

41. Yang, H., Xia, Y., Qiu, J., \& Zhang, J. (2010). Filtering for a class of discrete-time systems with time-delays via delta operator approach. International Journal of Systems Science, 41(4), 423-433. 